

TEXAS TECH UNIVERSITY

Libraries"

\title{
MODEL PREDICTIONS FOR ATMOSPHERIC AIR BREAKDOWN BY RADIO- FREQUENCY EXCITATION IN LARGE GAPS
}

\section{The Texas Tech community has made this publication openly available. Please share how this access benefits you. Your story matters to us.}

\begin{tabular}{|c|c|}
\hline Citation & $\begin{array}{l}\text { H. Nguyen, J. Mankowski, J. C. Dickens, A. A. Neuber, and R. P. Joshi, } \\
\text { "Model Predictions for Atmospheric Air Breakdown by Radio- } \\
\text { Frequency Excitation in Large Gaps," Physics of Plasmas 24, } 073505 \\
\text { (2017). https://doi.org/10.1063/1.4990699 }\end{array}$ \\
\hline Citable Link & https://hdl.handle.net/2346/86911 \\
\hline Terms of Use & $\begin{array}{l}\text { This article may be downloaded for personal use only. Any other } \\
\text { use requires prior permission of the author and AIP Publishing. }\end{array}$ \\
\hline
\end{tabular}




\section{Model predictions for atmospheric air breakdown by radio-frequency excitation in large gaps}

Cite as: Phys. Plasmas 24, 073505 (2017); https://doi.org/10.1063/1.4990699

Submitted: 14 May 2017 . Accepted: 14 June 2017 . Published Online: 29 June 2017

(D) H. K. Nguyen, (D) J. Mankowski, J. C. Dickens, (D) A. A. Neuber, and R. P. Joshi

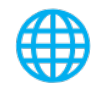

\section{ARTICLES YOU MAY BE INTERESTED IN}

Fundamental study of DC and RF breakdown of atmospheric air

Physics of Plasmas 26, 123512 (2019); https://doi.org/10.1063/1.5119152

Scaling laws for AC gas breakdown and implications for universality

Physics of Plasmas 24, 104501 (2017); https://doi.org/10.1063/1.4998245

Atmospheric-pressure gas breakdown from 2 to $100 \mathrm{MHz}$

Applied Physics Letters 93, 221505 (2008); https://doi.org/10.1063/1.3043449 


\title{
Model predictions for atmospheric air breakdown by radio-frequency excitation in large gaps
}

\author{
H. K. Nguyen, J. Mankowski, J. C. Dickens, A. A. Neuber, and R. P. Joshi ${ }^{\text {a) }}$ \\ Department of Electrical and Computer Engineering, Texas Tech University, Lubbock, Texas 79409, USA
}

(Received 14 May 2017; accepted 14 June 2017; published online 29 June 2017)

\begin{abstract}
The behavior of the breakdown electric field versus frequency (DC to $100 \mathrm{MHz}$ ) for different gap lengths has been studied numerically at atmospheric pressure. Unlike previous reports, the focus here is on much larger gap lengths in the $1-5 \mathrm{~cm}$ range. A numerical analysis, with transport coefficients obtained from Monte Carlo calculations, is used to ascertain the electric field thresholds at which the growth and extinction of the electron population over time are balanced. Our analysis is indicative of a U-shaped frequency dependence, lower breakdown fields with increasing gap lengths, and trends qualitatively similar to the frequency-dependent field behavior for microgaps. The low frequency value of $\sim 34 \mathrm{kV} / \mathrm{cm}$ for a $1 \mathrm{~cm}$ gap approaches the reported DC Paschen limit. Published by AIP Publishing. [http://dx.doi.org/10.1063/1.4990699]
\end{abstract}

\section{INTRODUCTION}

Gas breakdown under radio frequency $(\mathrm{RF})$ conditions is an important topic, and numerous studies have been reported in the literature. ${ }^{1-6}$ On the commercial side, applications of such breakdown under AC conditions include etching, ${ }^{7,8}$ thinfilm deposition, ${ }^{9}$ pollution control involving ozone depletion substances and volatile organic compounds, ${ }^{10}$ biomedical applications, ${ }^{11}$ pumping of gas lasers, ${ }^{12}$ etc. The use of atmospheric pressure plasmas greatly expands the scope and utility, and has prompted developments of numerous novel atmospheric pressure plasma sources including the atmospheric pressure plasma jet ${ }^{13}$ and the cold plasma torch. ${ }^{14}$ However, the aspect of RF breakdown at atmospheric pressures has not been well studied, in part, because sources capable of delivering power at hundreds of kilowatt to megawatt levels under at least long-pulse conditions are required, and these are not well developed. Gyrotrons are one of the few sources capable of delivering such power levels, though recent developments in relativistic magnetrons have also led to significant increases in the power output and efficiencies. ${ }^{15-17}$ Ongoing progress towards high output power will make gas breakdown issues quite important in future RF and millimeter wave systems. This topic could also be germane to atmospheric propagation and the prospect for large area plasma generation.

Air breakdown under high frequency conditions has been studied for over a century, and theoretical models are well described in a number of texts. ${ }^{18-20}$ However, the experimental data and analysis available in the literature have generally focused on the Pachen-like behavior relating the breakdown field $\left(E_{b r}\right)$ to the gas pressure $(p) \cdot{ }^{21-24}$ Very few reports have probed the $E_{b r}$ behavior in response to AC fields as a function of electrode separation in large gaps. The scant literature concerning breakdown in this regime has typically focused on much smaller gaps. ${ }^{25,26}$ Here, we specifically probe the behavior of $E_{b r}$ versus frequency in atmospheric air for gap lengths in the $1-5 \mathrm{~cm}$ regime. Our interest is in large gaps, and eventually

\footnotetext{
${ }^{a)}$ Author to whom correspondence should be addressed: ravi.joshi@ttu.edu
}

larger volume plasmas driven by a need to test concepts and obtain benchmarks for use towards ionospheric plasma generation by RF sources.

\section{MODEL DETAILS}

Unlike dc discharges for which the flow of ions to the cathode may play a role at small overvoltages, the breakdown behavior at higher frequencies is controlled mainly by electron transport. Treatment of RF breakdown had been considered by Kihara ${ }^{27}$ based on the Boltzmann theory that included bulk ionization, periodic electron drift, and diffusion. In the RF regime, the positional oscillation amplitude of the electrons due to the applied fields is small relative to the discharge dimensions, making large energy gains or highly nonlinear transport quite unlikely. Consequently in this scenario of RF frequencies, applying a drift-diffusion model [rather than a full Monte Carlo (MC) technique, which is more time-consuming] for probing the electron transport behavior would be valid. The full MC technique can be appropriate for high frequencies or small gaps since the overall simulation time (a few cycles for high frequencies, or short transit times for narrow gaps) would not be computationally prohibitive. However, the MC method becomes numerically intractable for low frequency excitations or for larger gaps. Here, under atmospheric conditions (that guarantee large scattering and local quasi-equilibrium), the drift-diffusion approach was chosen, and MC calculations were used to accurately obtain the transport parameters. Also, movement of ions is severely limited due to their large mass as compared to electrons. Hence, ions were treated as being virtually immobile. Furthermore, for calculations of the breakdown threshold where one is at the verge of a selfsustained plasma, the electron and ion densities can be assumed to be relatively modest. Under this scenario, the gas remains weakly ionized (i.e., $n_{e} \ll n_{n}$, where $n_{e}$ and $n_{n}$ are the free electron and neutral densities, respectively), and so electron-electron, ion-ion, and electron-ion scattering can all be neglected. Also, unlike microgaps where field 
emission due to cathode electron tunneling can modify the characteristic behavior, ${ }^{28}$ this and other nanoscale or quantum aspects were ignored here. Deviations from the Paschen behavior in microgaps were first reported in Torres and Dhariwal's seminal work ${ }^{29}$ and later shown to arise from electron field emission. ${ }^{30}$ Studies also demonstrated the presence of positive ions near the cathode to make additive contributions to such emission and associated reductions in breakdown voltage. In the present scenario such high fields do not arise due to longer gaps, nor would ions be very likely to be driven close to the electrodes under the oscillating field conditions.

To determine gas breakdown from the standpoint of a simple model, it is useful to examine the variation of electron concentrations over time. Net growth in electron density over time signals a scenario wherein one is past the breakdown point. Similarly, at the breakdown threshold, the increase the net density would be balanced by electron losses at the surfaces and through attachment. Thus, a possible approach to determining the atmospheric pressure threshold field for a given length between electrode plates is to carry out drift-diffusion analyses to obtain the electron density evolution $n(x, t)$. A near steady, time-independent value of the total population in the presence of the RF excitation of amplitude $E_{0}$ signals the breakdown condition.

Simplifications inherent in the present scheme are as follows: (a) absence of secondary electron emission that might occur at the electrodes. Under the conditions of interest involving the oscillating fields and high pressure (with its high scattering rate), it is reasonable to assume that electrons are unlikely to impinge on the electrodes with the high energy required for secondary emission. Thus, only bulk ionization within the gas was considered. Similarly, electron emission due to impact of ions at the electrodes was ignored, given the improbable chance that more massive ions would possess sufficiently high impact energies. (b) High-energy ion-neutral collisions can also be ignored under these RF excitation conditions. Besides, ion densities are anyway low around the breakdown threshold. (c) This treatment assumes a continuum, implying that the electronic mean-free paths are much smaller than the electrode spacing $L$. For the atmospheric (high pressure) conditions of interest here, this requirement is easily satisfied. (d) Finally, the electron production due to photo-ionization, while important for streamer discharges at high over voltages, ${ }^{31}$ has been discounted.

The transport parameters for the drift-diffusion equation were calculated from the Monte Carlo method. The latter ensured that nonlinear dependencies on the driving electric field, phase shifts, and dynamic changes in the electron distribution function which control the transport parameters were adequately included. Field-dependent ionization rates were used to augment electron densities as a function of time and position. Losses at the boundaries were included through outflows based on the continuity equation. For a onedimensional geometry involving parallel electrodes separated by a distance $L$, the rate equation for electron density $n(x, t)$ takes the form

$$
\partial n / \partial t=G+\left[R_{i}(E)-R_{\text {loss }}(E)\right] n-\partial\left(F_{n}\right) / \partial x
$$

with

$$
F_{n}=n v_{n d r}(E)-D_{n} \partial n / \partial x,
$$

where $D_{n}=$ electron diffusion coefficient, $G=$ background generation due to cosmic radiation, photons, etc., $R_{i}=$ ionization rate which equals the product of the ionization coefficient $(\alpha)$ and the electron drift velocity, $R_{\text {loss }}=$ loss rate, $E=$ the applied RF field with peak amplitude $E_{0}$, while $\omega$ is the RF angular frequency and $F_{n}$ represents the electron flux in response to the applied electric field. In our numerical approach, the losses were simply computed numerically from the outflow of charge at the boundaries due to drift and diffusion. A one-dimensional Poisson solver was included to account for space-charge effects. Several parameters that influence electron density and its dynamics, such as $R_{i}(E)$, $R_{\text {loss }}(E)$, and the drift velocity $v_{n d r}(E)$, all depend implicitly on the local electric field. Strictly, even the diffusion coefficient $D_{n}$, which is related to either the velocity autocorrelation function or the mean-square displacement via the Green-Kubo relation, ${ }^{32,33}$ can be a function of the electric field.

Here, the Monte Carlo approach, as reported and discussed in detail by our group elsewhere, ${ }^{34,35}$ was used for computations of the transport parameters. The standard scattering mechanisms for both elastic and inelastic collisions between electrons and both nitrogen and oxygen were implemented. ${ }^{36,37}$ The cross-sections were taken from Phelps and Pitchford $^{38}$ and Itikawa et al. ${ }^{39,40}$ While the field dependent drift velocities were directly obtained from the MC, the first Townsend coefficient $\alpha$ was calculated from the ratio of the ionization rate and the electron drift velocity $\left(v_{n d r}\right)$, averaged over the entire ensemble of particles at each time step. Finally, the field-dependent diffusion coefficient $D(E)$ is given in terms of the velocity autocorrelation function $\psi(E, t)$ as ${ }^{32,33}$

$$
D(E)=(1 / 3) \int_{0}^{\infty} d t \psi(E, t)
$$

with

$$
\psi(E, t)=\langle v(E, t) \cdot v(E, 0)\rangle .
$$

The $\psi(E, t)$ involves a dot product over the electronic velocities for the $i$ th particle at time " $t$ " with its corresponding value at an earlier time. The frequency dependence for the diffusion follows from its relation to the noise power spectral density associated with the fluctuations and is given as: ${ }^{41}$ $D(\omega, E)=S(\omega) / 4=(1 / 3) \int_{0}^{\infty} d t \psi(E, t) \cos (\omega t)$.

\section{RESULTS AND DISCUSSION}

Monte Carlo results obtained for the electron drift velocity are shown in Fig. 1 as a function of the electric field. Although simulations for air were carried out, Fig. 1 shows the values for a nitrogen ambient simply to facilitate easy comparisons and validation. Also shown are the experimental data points, with values taken from Hasegawa et $\mathrm{al}^{42}$ Wedding and coworkers, ${ }^{43}$ Roznerski, ${ }^{44}$ Fletcher and Reid, ${ }^{45}$ and Blevin and Kelly ${ }^{46}$ for nitrogen. The Monte Carlo results seem to be in very good agreement with the data. The maximum deviation 


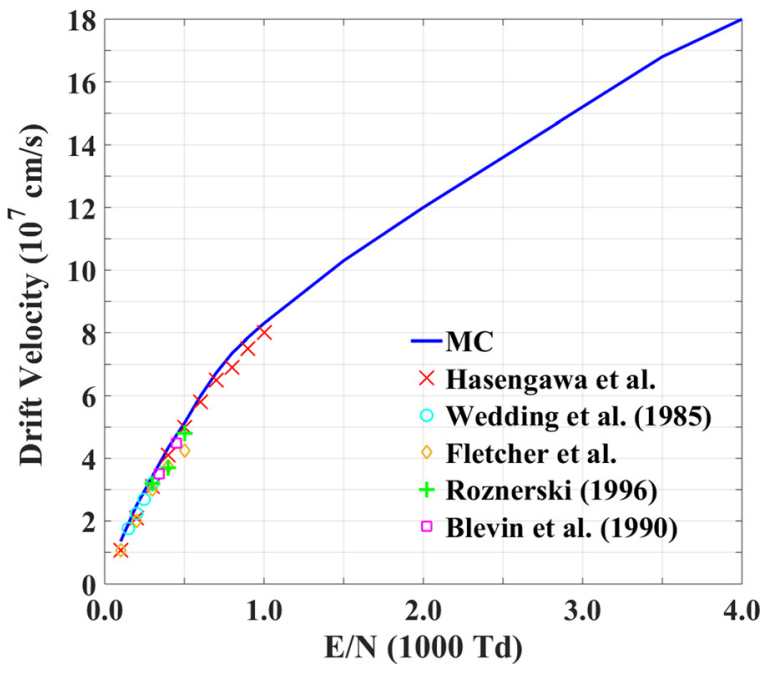

FIG. 1. Results of the electron drift velocity as a function of electric field in nitrogen at atmospheric pressure. The reported experimental data are also shown for comparison.

was at the highest electric field and the result predicted by the simulation was within $5 \%$ of the experimental data. The diffusion coefficient was obtained from calculations of the velocity autocorrelation function at different electric fields. A representative plot of $\psi(t)$ at a $E / N$ value of $90 \mathrm{Td}$ is shown in Fig. 2(a). As might be expected, the value starts from unity and decays towards zero, with small oscillations at longer times. The diffusion coefficient obtained from a numerical integration of Fig. 2(a) yielded a value of $0.0755 \mathrm{~m}^{2} / \mathrm{s}$. Fielddependent values for the diffusion coefficient $D$ obtained from such velocity autocorrelation results are shown in Fig. 2(b). The magnitudes and trend are in close agreement with a report by Hagelaar and Pitchford. ${ }^{47}$

Field-dependent values of the first Townsend coefficient $(\alpha)$ obtained from our Monte Carlo simulations, with direct comparisons to experimental data, are shown in Fig. 3. The results show close agreement with the reports by Legler, ${ }^{48}$ and Pitchford and Phelps. ${ }^{49}$ A maximum deviation of $0.25 \times$ $10^{-16} \mathrm{~cm}^{2}$ for $\alpha / N$ was obtained for a reduced field $(E / N)$ of $3000 \mathrm{Td}$ and represented about a $6.5 \%$ deviation from the Pitchford data. For convenient use of the transport parameters in the drift-diffusion approach, analytic expressions were obtained from suitable curve-fitting of the MC results. For example, the expression (in CGS units) of $\alpha(E)=1.4764 \times$ $10^{-4} \exp \left(-3.5 \times 10^{3} / \mathrm{E}\right)$ resulted for the ionization coefficient $\alpha(E)$ in terms of the electric field $E$. It is important to note, as was discussed by Stephens et al. ${ }^{31}$ and elsewhere, that the electron temperature follows temporal changes in the electric field within a few tens of picoseconds, primarily due to the high collision frequencies at atmospheric pressures. Since the $\mathrm{RF}$ frequencies of interest are in the $100 \mathrm{MHz}$ range and below, it is reasonable to consider quasistatic electric field conditions for the electron temperature and the electron energy distribution function (EEDF). In other words, the transport parameters calculated with a static electric field track the RF field amplitude in the drift-diffusion simulation.

The results obtained from simulations are shown in Fig. 4. The time- and spatial-evolution of the electron densities in
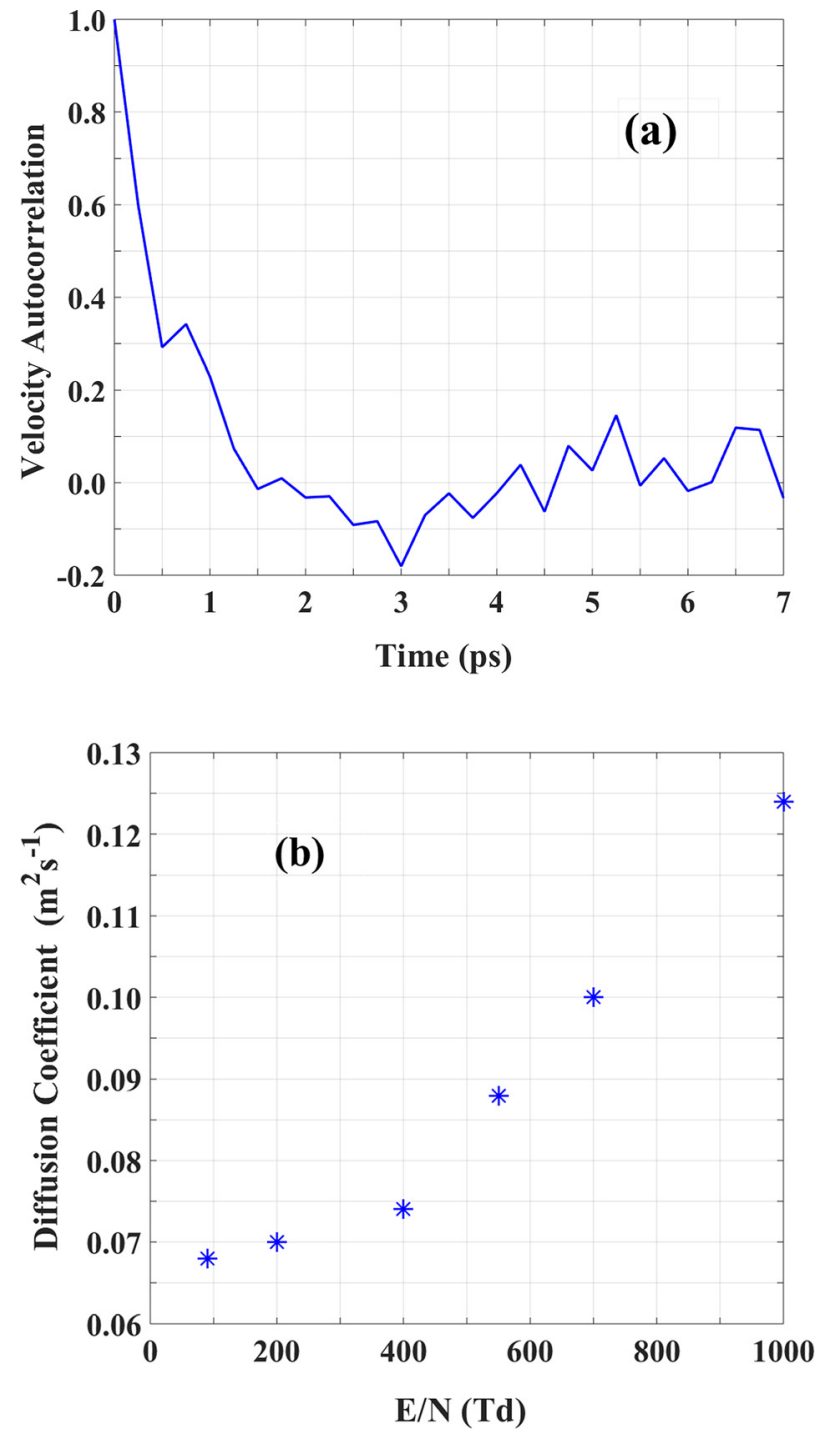

FIG. 2. (a) Simulated results for the velocity autocorrelation function at E/ $\mathrm{N}=90$ Td. (b) Calculated diffusion coefficient versus electric field in nitrogen at atmospheric pressure for $300 \mathrm{~K}$.

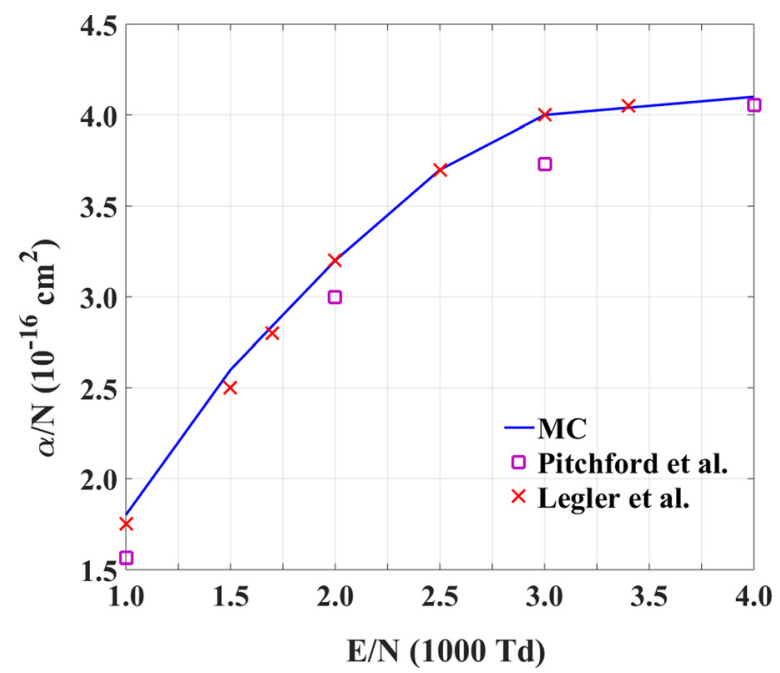

FIG. 3. Predicted field dependence of the electron impact ionization coefficient $(\alpha)$ as obtained from Monte Carlo simulations. Numerous experimental data points are shown for comparison. 

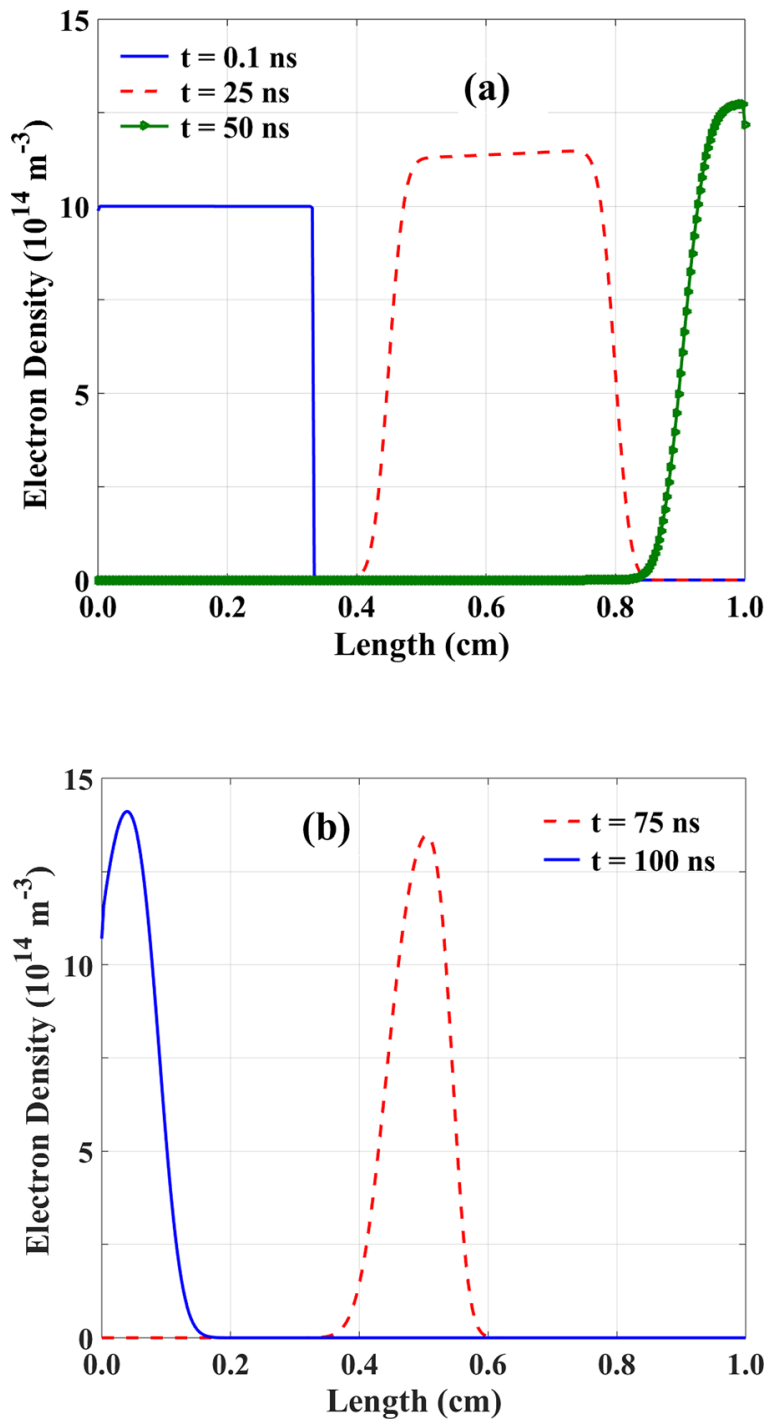

FIG. 4. Simulations for the time- and spatial-evolution of the electron density in the parallel plate air gap for a $1 \mathrm{~cm}$ gap length, an applied frequency of $10 \mathrm{MHz}$, and $E_{0}=28 \mathrm{kV} / \mathrm{cm}$. (a) Initial snapshots at three different times of $0.1 \mathrm{~ns}, 25 \mathrm{~ns}$, and $50 \mathrm{~ns}$, showing the electron swarm moving to the right starting from an initial rectangular distribution. (b) Snapshots of the electron profile $n(x, t)$ at 75 and 100 ns with the electric field now reversed.

a $1 \mathrm{~cm}$ air gap were simulated at an applied frequency of $100 \mathrm{MHz}$ and $E_{0}=28 \mathrm{kV} / \mathrm{cm}$ [i.e., electric field $E(t)=E_{0}$ $\sin (\omega t)]$. Figure 4(a) shows the snapshots of the electron density profile $n(x, t)$ at three different times of $0.1 \mathrm{~ns}, 25 \mathrm{~ns}$, and $50 \mathrm{~ns}$. The electron swarm was taken to have an initial rectangular distribution over the left-third of the air gap between the parallel plates. Upon application of a negative field, a gradual movement to the right can be seen. Figure 4(b) shows the snapshots of the electron profile $n(x, t)$ at longer times of 75 and $100 \mathrm{~ns}$, with the electric field now reversed. The losses at the electrodes are obvious, as the rectangular shape gets shortened and also changes over to a smoother profile due to diffusive contributions.

The time evolution of the entire electron swarm at different applied electric field magnitudes is shown in Fig. 5 for a gap length of $1 \mathrm{~cm}$ and an operating frequency of $10 \mathrm{MHz}$. The initial electron density distribution was taken to be uniform. While Fig. 5(a) shows the entire temporal span, the
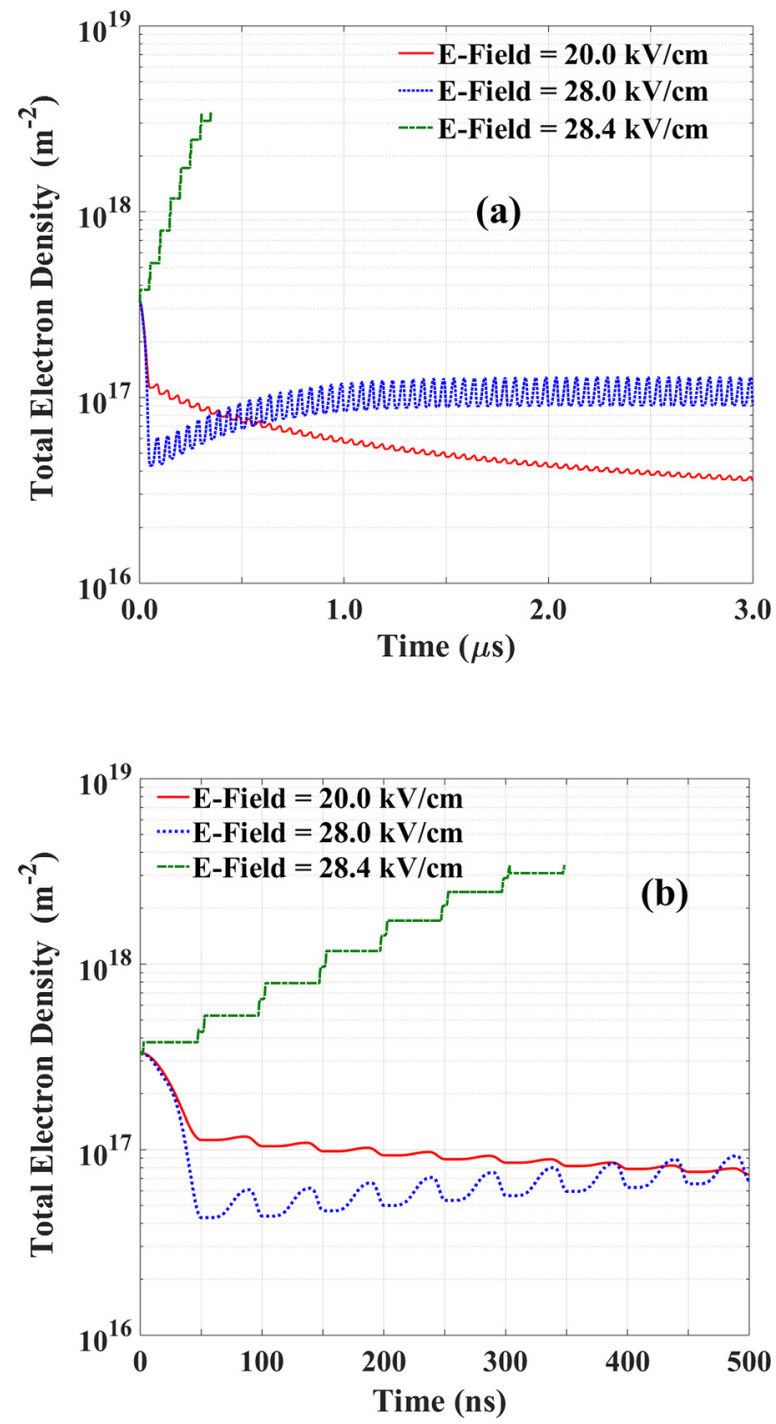

FIG. 5. (a) Drift-diffusion simulation results for the time evolutionary behavior of the electron population within the parallel plates at three different applied field amplitudes $E_{O}$ of $20 \mathrm{kV} / \mathrm{cm}, 28 \mathrm{kV} / \mathrm{cm}$, and $28.4 \mathrm{kV} / \mathrm{cm}$. A gap length of $1 \mathrm{~cm}$ and an operating frequency of $10 \mathrm{MHz}$ were used for the calculations. (b) Expanded version of Fig. 5(a) covering a shorter, initial time.

initial portion of the total electron density evolution is shown in Fig. 5(b) for clarity. At the lowest $20 \mathrm{kV} / \mathrm{cm}$ electric field value, the electron population is seen to show a slow but monotonic decrease over time. This clearly signals a nonself-sustaining situation. The minor oscillations are simply indicative of the interplay between periods of growth fueled by volume ionization when the swarm position is situated in regions removed from the end plates, and times when population reductions occur due to boundary absorption. At the field amplitude of $28.4 \mathrm{kV} / \mathrm{cm}$, a prominent growth is predicted. In fact, due to a near exponential increase, only a small fraction of the entire time span is shown for the $28.4 \mathrm{kV} / \mathrm{cm}$ case. Finally, the $E_{0}=28 \mathrm{kV} / \mathrm{cm}$ curve attains a near steady state where growth is balanced by losses, and a steady-state is evident. This field amplitude thus represents the breakdown threshold for the $10 \mathrm{MHz}$ signal.

The results for the breakdown characteristic versus frequency are shown in Fig. 6. Two plots corresponding to 


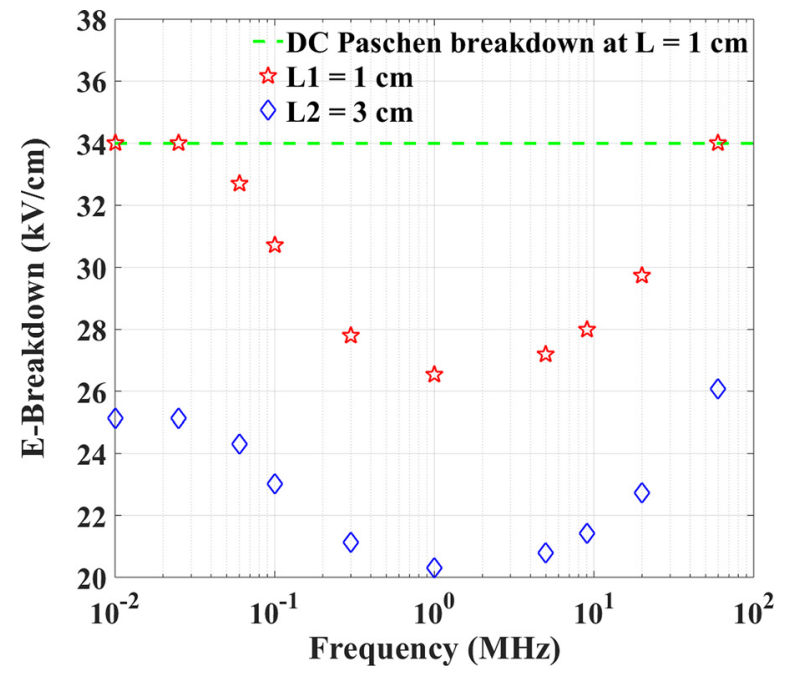

FIG. 6. Simulation results obtained for the breakdown field strength versus operating frequency for two different gap lengths. The low frequency value of $\sim 34 \mathrm{kV} / \mathrm{cm}$ for a $1 \mathrm{~cm}$ gap approaches the reported DC Paschen limit.

electrode separation distances $L$ of $1 \mathrm{~cm}$ and $3 \mathrm{~cm}$ were obtained. Roughly, a U-shaped curve results and is in line with qualitative expectations. The minimum corresponds to the situation where electrons continue to oscillate rapidly, while ions are almost frozen in space. However, unlike the case of micro-gaps, the physics here does not totally change over from a diffusion-controlled process to boundarydominated behavior. Also, at very low frequencies, our values for the $1 \mathrm{~cm}$ gap length agree fairly well with the DC Paschen breakdown value ${ }^{25}$ as indicated by the dashed line in Fig. 6, and the boundary effects play a role. Also, $E_{b r}$ has a very weak dependence on the frequency in this domain, a behavior similar to the trends seen in microgaps for the boundary-dominated regime. At high frequencies, on the other hand, the electrons no longer gain sufficient energy from the field, and so contribute increasingly less to ionizing collisions. Hence, ever larger electric fields are needed to reach the breakdown threshold. Overall, about a $20 \%$ reduction in breakdown electric field over the DC case is observed for either gap around the 1 to $2 \mathrm{MHz}$ frequency regime. The current prediction is qualitatively in agreement with a previous report of radiofrequency breakdown by Walsh et al. ${ }^{50}$ in the $2-100 \mathrm{MHz}$ range. Their experimental data showed the breakdown voltage to reduce initially with increasing frequency due to diminishing contribution of drift-dominated electron wall losses, followed by an increase at higher frequencies. A similar qualitative trend was reported by Uchida et al. ${ }^{51}$ for frequencies ranging from few tens of megahertz to $100 \mathrm{MHz}$. Finally, the breakdown voltages for the larger $3 \mathrm{~cm}$ gap are predicted to be lower than for the $1 \mathrm{~cm}$ case for all frequencies. Physically, this arises due to a decrease in the loss terms since electrons are less likely to reach either boundary within a given frequency cycle for a longer gap. However, the current technique may not work well for simulations of plasma dynamics well beyond threshold conditions as many of the assumptions outlined earlier may no longer hold true. As a final comment, besides electron emission, ${ }^{28,30,52}$ field-dependent secondary emission may be another process that might alter the breakdown characteristic somewhat, impart a field and frequency dependence, and may need to be considered for completeness. Secondary electron emission can be facilitated either by incoming ions $\left(\gamma_{\mathrm{se}}\right)$, incident electrons, or by a photon-generated event. ${ }^{31}$ The latter process might play an increasingly important role at high frequencies, since the ions (and ultimately the electrons) might not be able to move much. The photon process, on the other hand, involves electronic excitation of neutral atoms, followed by the decay of meta-stable excited neutrals leading to photon emission. Photoemission can then result from some fraction of such photons striking the electrodes. The relative significance of such contributions can be expected to depend on the applied field and frequency, and would conceivably lower the breakdown threshold somewhat. Such frequency dependent changes in the driving mechanisms were recently discussed in a slightly different context of high frequency discharges by Lee et al., ${ }^{52,53}$ though the photon mechanism was not included. These aspects will be analyzed and discussed elsewhere.

\section{CONCLUSIONS}

In conclusion, we have probed the breakdown dependence in air at atmospheric pressures subject to AC excitation. The frequency changes over orders of magnitude were analyzed. Most previous studies of RF breakdown have focused on the field dependence on pressure. From our simulations, a U-shaped frequency dependent breakdown field characteristic was obtained; with the low frequency value of $\sim 34 \mathrm{kV} / \mathrm{cm}$ for a $1 \mathrm{~cm}$ gap approaching the reported DC Paschen limit. The results can also serve as a useful guide to determine the safe operating voltage limits at various frequencies in atmospheric air. The voltage ranges at the higher frequencies, however, may be practically difficult to attain based on current technology.

\section{ACKNOWLEDGMENTS}

This work was supported in part by the Air Force Office of Scientific Research under Award No. FA9550-14-1-0019.

\footnotetext{
${ }^{1}$ A. D. MacDonald, Microwave Breakdown in Gases (Wiley, New York, 1966).

${ }^{2}$ Y. P. Raizer, Gas Discharge Physics (Springer, Berlin, 1997).

${ }^{3}$ A. V. Gurevich, N. D. Borisov, and G. M. Milikh, Physics of Microwave Discharges (Gordon and Breach, Amsterdam, 1997).

${ }^{4}$ Y. Hidaka, E. M. Choi, I. Mastovsky, M. A. Shapiro, J. R. Sirigiri, and R. J. Temkin, Phys. Rev. Lett. 100, 035003 (2008).

${ }^{5}$ Y. Oda, K. Komurasaki, K. Takahashi, A. Kasugai, and K. Sakamoto, J. Appl. Phys. 100, 113307 (2006).

${ }^{6}$ H. B. Smith, C. Charles, and R. W. Boswell, Phys. Plasmas 10, 875 (2003).

${ }^{7}$ D. J. Economou, J. Phys. D: Appl. Phys. 47, 303001 (2014).

${ }^{8}$ V. M. Donnelly and A. Kornblit, J. Vac. Sci. Technol. A 31, 050825 (2013).

${ }^{9}$ C. Strobel, B. Leszczynska, U. Merkel, J. Kuske, D. D. Fischer, M. Albert, J. Holovský, S. Michard, and J. W. Bartha, Sol. Energy Mater. Sol. Cells 143, 347 (2015).

${ }^{10}$ J. S. Chang, Sci. Technol. Adv. Mater. 2, 571 (2001).

${ }^{11}$ M. Laroussi and T. Akan, Plasma Process. Polym. 4, 777 (2007).

${ }^{12}$ Y. P. Raizer, M. N. Shneider, and N. A. Yatsenko, Radio-Frequency Capacitive Discharges (CRC Press, Florida, USA, 1995).
} 
${ }^{13}$ J. Park, I. Henins, H. W. Herrmann, G. S. Selwyn, J. Y. Jeong, R. F. Hicks, D. Shim, and C. S. Chang, Appl. Phys. Lett. 76, 288 (2000).

${ }^{14}$ C. Hoffmann, C. Berganza, and J. Zhang, Med. Gas Res. 3, 21 (2013).

${ }^{15}$ W. Li and Y. G. Liu, J. Appl. Phys. 106, 053303 (2009).

${ }^{16}$ V. B. Neculaes, M. C. Jones, R. M. Gilgenbach, Y. Y. Lau, J. W. Luginsland, B. W. Hoff, W. M. White, N. M. Jordan, P. Pengvanich, Y. Hidaka, and H. L. Bosman, IEEE Trans. Plasma Sci. 33, 94 (2005).

${ }^{17}$ W. Li, Y. G. Liu, T. Shu, H. W. Yang, Y. W. Fan, C. W. Yuan, and J. Zhang, Phys. Plasmas 19, 013105 (2012).

${ }^{18}$ S. C. Brown, Basic Data of Plasma Physics (MIT Press, Cambridge, MA, 1959).

${ }^{19}$ Y. P. Raizer, Gas Discharge Physics (Springer-Verlag, Berlin, 1997).

${ }^{20}$ F. M. Penning, Electrical Discharges in Gases, translated from the Dutch by A. F. Monypenny (Cleaver-Hume, London, 1957).

${ }^{21}$ A. V. Lisovskiy and V. D. Yegorenkov, J. Phys. D 31, 3349 (1998).

${ }^{22}$ V. A. Lisovskiu and V. D. Yegorenkov, J. Phys. D 27, 2340 (1994).

${ }^{23}$ F. Hohn, W. Jacob, R. Beckmann, and R. Wilhelm, Phys. Plasmas 4, 940 (1997).

${ }^{24}$ Z. L. Petrović, J. Sivoš, M. Savić, N. Škoro, M. R. Rađenović, G. Malović, S. Gocić, and D. Marić, J. Phys.: Conf. Ser. 514, 012043 (2014).

${ }^{25}$ E. Nasser, Fundamentals of Gaseous Ionization and Plasma Electronics (Wiley-Interscience, New York, 1971).

${ }^{26}$ A. Semnani, A. Venkattraman, A. A. Alexeenko, and D. Peroulis, Appl. Phys. Lett. 103, 063102 (2013).

${ }^{27}$ T. Kihara, Rev. Mod. Phys. 24, 45 (1952).

${ }^{28}$ P. Rumbach and D. B. Go, J. Appl. Phys. 112, 103302 (2012).

${ }^{29}$ J. M. Torres and R. S. Dhariwal, Nanotechnology 10, 102 (1999).

${ }^{30}$ D. B. Go and A. Venkattraman, J. Phys. D 47, 503001 (2014).

${ }^{31}$ J. Stephens, A. Fierro, S. Beeson, G. Laity, D. Trienekens, R. P. Joshi, J. Dickens, and A. Neuber, Plasma Sources Sci. Technol. 25, 025024 (2016).

${ }^{32}$ M. S. Green, J. Chem. Phys. 22, 398 (1954).

${ }^{33}$ R. Kubo, J. Phys. Soc. Jpn. 12, 570 (1957).

${ }^{34}$ A. Fierro, J. C. Dickens, and A. N. Neuber, Phys. Plasmas 21, 123504 (2014).
${ }^{35}$ A. Fierro, J. Stephens, S. Beeson, J. Dickens, and A. Neuber, Phys. Plasmas 23, 013506 (2016).

${ }^{36}$ J. P. Verboncoeur, Plasma Phys. Controlled Fusion 47, A231-A260 (2005).

${ }^{37}$ J. P. Boeuf and E. Marode, J. Phys. D 15, 2169 (1982).

${ }^{38}$ A. V. Phelps and L. C. Pitchford, JILA Information Report No. 26 (University of Colorado, Boulder, CO, 1985); A. V. Phelps and L. C. Pitchford, Phys. Rev. A 31, 2932 (1985).

${ }^{39}$ Y. Itikawa, M. Hayashi, A. Ichimura, K. Onda, K. Sakimoto, K. Takayanagi, M. Nakamura, H. Nishimura, and T. Takayanagi, J. Phys. Chem. Ref. Data 15, 985 (1986).

${ }^{40}$ Y. Itikawa, J. Phys. Chem. Ref. Data 38, 1 (2009).

${ }^{41}$ R. Fauquembergue, J. Zimmermann, A. Kaszynski, and E. Constant, J. Appl. Phys. 51, 1065 (1980).

${ }^{42}$ H. Hasegawa, H. Date, M. Shimozuma, K. Yoshida, and H. Tagashira, J. Phys. D 29, 2664 (1996).

${ }^{43}$ A. B. Wedding, H. A. Blevin, and J. Fletcher, J. Phys. D 18, 2361 (1985).

${ }^{44}$ W. Roznerski, J. Phys. D 29, 614 (1996).

${ }^{45}$ J. Fletcher and I. D. Reid, J. Phys. D 13, 2275 (1980).

${ }^{46}$ H. A. Blevin and L. J. Kelly, Nonequilibrium Effects in Ion and Electron Transport, edited by J. W. Gallagher, D. F. Hudson, E. E. Kunhardt, and R. J. van Brunt (Plenum Press, New York, 1990), pp. 67-81.

${ }^{47}$ G. J. M. Hagelaar and L. C. Pitchford, Plasma Sources Sci. Technol. 14, 722 (2005)

${ }^{48}$ W. Legler, Z. Phys. 173, 169 (1963).

${ }^{49}$ L. C. Pitchford and A. V. Phelps, Phys. Rev. A 25, 540 (1982).

${ }^{50}$ J. L. Walsh, Y. T. Zhang, F. Iza, and M. G. Kong, Appl. Phys. Lett. 93, 221505 (2008).

${ }^{51}$ G. Uchida, K. Takenaka, A. Miyazaki, and Y. Setsuhara, J. Nanosci. Nanotechnol. 15, 2192 (2015).

${ }^{52}$ M. U. Lee, J. Lee, J. K. Lee, and G. S. Yun, Plasma Sources Sci. Technol. 26, 034003 (2017).

${ }^{53}$ M. U. Lee, J. Lee, G. S. Yun, and J. K. Lee, Eur. Phys. J. D 71, 94 (2017). 